\title{
Expansão dentoalveolar com aparelho quadrihélice: um relato de caso
}

\author{
Posterior crossbite treated with quad helix: a case report
}

\begin{abstract}
RESUMO
Introdução: A mordida cruzada posterior é definida como uma relação transversal inadequada entre maxila e mandíbula. Pode ter envolvimento esquelético, funcional ou dentoalveolar. Objetivo: Avaliar a eficácia do aparelho quadrihélice na correção da mordida cruzada posterior dentoalveolar em indivíduo Classe I de Angle. Relato do Caso: Paciente de 15 anos e 7 meses de idade, sexo masculino, foi tratado com aparelho quadrihélice. $\mathrm{O}$ aparelho foi ativado em média uma vez por mês e o tempo de tratamento foi de 8 meses. Foram avaliados parâmetros clínicos pré e pós expansão da maxila. A análise dos modelos em gesso obtidos antes da instalação do aparelho e após o descruzamento da mordida permitiu avaliar a discrepância de modelos, a largura dos arcos superior e inferior na região de caninos, $1^{\mathrm{O}}$ pré-molares, $2^{\circ}$ prémolares e $1^{\underline{0}}$ molares, sobremordida e sobressaliência. Resultados: A distância entre 13-23 manteve-se constante de T0 para T1; a distância entre 14-24, 15-25, 16-26, 36-46 aumentaram de T0 para T1; e de 33-43, 34-44 e 35-45 diminuíram. O apinhamento antero-superior diminuiu de T0 $(8 \mathrm{~mm})$ para T1 $(7 \mathrm{~mm})$ e o antero-inferior diminuiu de T0 $(3 \mathrm{~mm})$ para T1 $(1 \mathrm{~mm})$. Sobressaliência diminuiu $0,5 \mathrm{~mm}$ e sobremordida aumentou 0,87mm. Conclusões: O aparelho quadrihélice foi eficaz na correção da mordida cruzada dentoalveolar unilateral.
\end{abstract}

Palavras-chave: Má Oclusão, Mordida cruzada, Técnica de Expansão Palatina

\begin{abstract}
Introduction: The posterior crossbite is defined as an unequal transversal relation between the jaws and it could be skeletal, functional or dentoalveolar. Objective: Evaluate the quad helix appliance in correcting the dentoalveolar posterior crossbite in an Angle's Class I patient. Case: A 15-years-old male patient was treated with quad helix appliance that was monthly activated. The treatment was finished in eight months. Clinical parameters were evaluated before (T0) and after (T1) maxillary expansion. The dental casts analysis showed the crowding; the upper and lower archs width in cuspid, bicuspids and tricuspids region; overbite and overjet. Results: The distance between 13-23 remained constant from T0 to T1; the distance between 14-24, 15-25, 16-26, 36-46 increased from T0 to T1; and from 33-43, 34-44 and 35-45 decreased. The anterior-upper crowding decreased from T0 $(-8 \mathrm{~mm})$ to T1 $(-7 \mathrm{~mm})$ and the anterior-lower decreased from T0 $(3 \mathrm{~mm})$ to T1 $(1 \mathrm{~mm})$. Overjet decreased $0.5 \mathrm{~mm}$ and overbite increased $0.87 \mathrm{~mm}$. Conclusions: The quad helix appliance was effective in the unilateral dentoalveolar crossbite's correction.
\end{abstract}

Uniterms: Malocclusion, Crossbite, Palatal Expansion Technique Introdução

\section{Débora Ok Pak ${ }^{1}$ \\ Anselmo Carmona de Lima \\ Nataly Nascimento Lemos da Silva ${ }^{2}$ \\ Renata Pilli Jóias ${ }^{3}$}

${ }^{1}$ DDS. Aluno do Curso de Especialização em

Ortodontia, Universidade Metodista de São

Paulo

2 Aluna do Curso de Odontologia, Universidade Metodista de São Paulo

${ }^{3}$ DDS, MsC, PhD. Professora do Curso de

Odontologia e do Curso de Especialização em

Ortodontia, Universidade Metodista de São

Paulo

Autor para correspondência:

Débora Ok Pak

Rua Porangaba, 566 - Jardim Nice - Santo

André/SP.

E-mail: debora.okpak@yahoo.com.br;

Tel (11) 977080802. 


\section{INTRODUÇÃO}

A mordida cruzada posterior é definida como uma relação transversal inadequa$\mathrm{da}^{1,2}$ entre maxila ${ }^{1}$ e mandibula $\mathrm{a}^{1,3}$ e há casos com envolvimento esquelético, funcional ou dentoalveolar ${ }^{3}$.

Mesmo com a eliminação dos fatores etiológicos, em menos de $16 \%$ dos casos observa-se autocorreção ${ }^{4}$, por isso é indicado que o tratamento seja realizado o quanto antes ${ }^{5}$. Geralmente, é associada à deficiência transversal da maxila que é resultado do crescimento assimétrico dos maxilares, da largura discrepante de ambos, perda prematura ou retenção prolongada de dentes deciduos, entre outros ${ }^{6}$.

Sua etiologia inclui fatores genéticos, ambientais e hábitos bucais deletérios ${ }^{6,7}$, sendo o hábito de sucção um dos principais fatores. Até os 4 anos de idade, em geral, não há risco de alteração grave na oclusão; a partir dos 5 anos, havendo permanência do hábito, diversas maloclusões poderão ser desenvolvidas ${ }^{8}$ e tornarão-se evidentes durante a dentição mista precoce ${ }^{9}$.

Dentre as inúmeras opções de aparelho, o quadrihélice é amplamente utilizado na correção das desarmonias transversais dentoalveolares ${ }^{2,5}$. Devido às suas várias formas de confecção e ativação, tornou-se útil não somente na sobrecorreção da discrepância transversal e sagital, mas também no tratamento das mordidas abertas anteriores. ${ }^{10,11}$

Considerando que o quadrihélice pode abreviar a fase de alinhamento e nivelamento do tratamento ortodôntico em até 4 a 5 meses quando comparado ao tratamento corretivo convencional, nesse relato de caso será avaliada sua eficácia na correção da mordida cruzada dentoalveolar previamente à mecânica ortodontica corretiva.

\section{RELATO DE CASO CLÍNICO}

Paciente G.F.W.L.S. 15 anos de idade, sexo masculino, compareceu a clínica de odontologia da Universidade Metodista de São Paulo com queixa principal de dentes tortos. O paciente apresentava simetria facial, selamento labial passivo, ângulo naso-labial obtuso e padrão mesio-facial (Figura 1). Análise cefalométrica (Tabela 1).

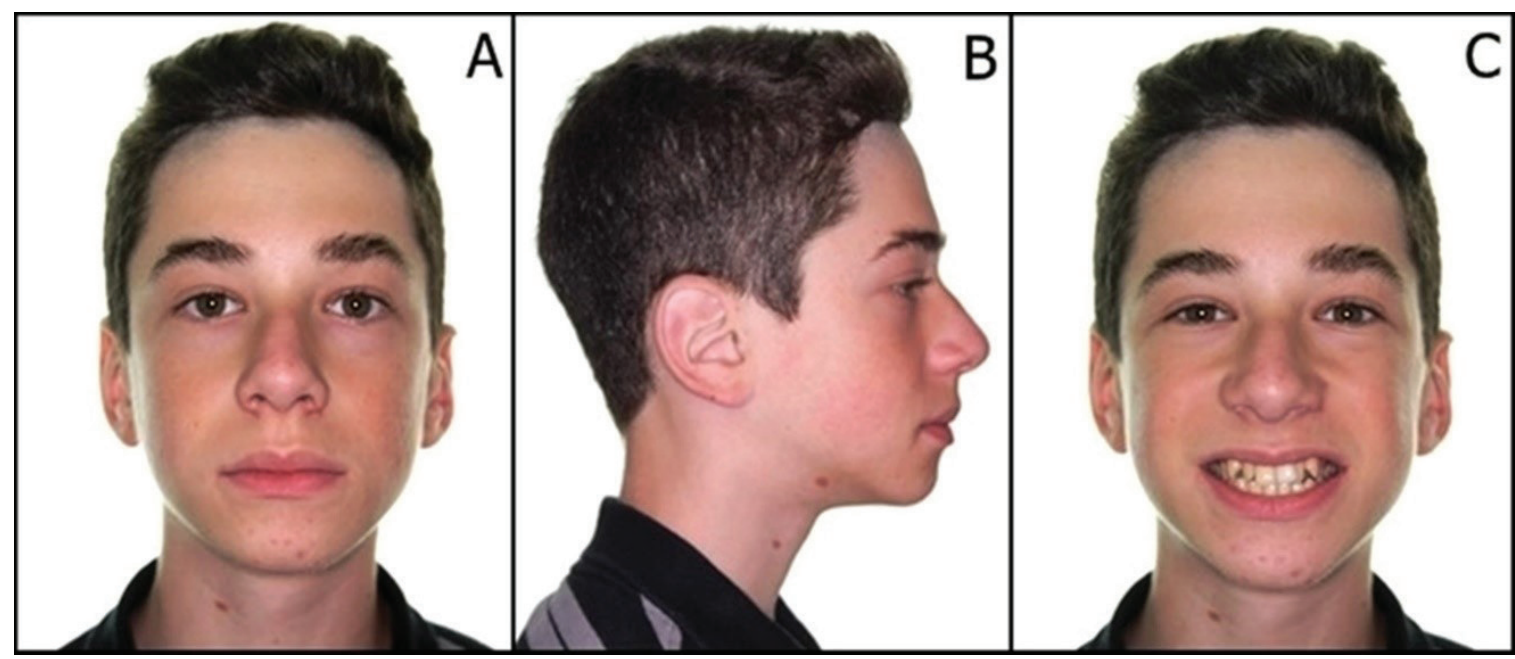

Figura 1: Fotos extra-bucais pré-tratamento (A-C). 
Tabela 1: Análises cefalométricas

\begin{tabular}{l|l}
\hline VERT & $-0,05$ \\
\hline FMA & $27,63^{\circ}$ \\
\hline Ângulo do eixo facial & $-6,27^{\circ}$ \\
\hline AFAI & $2,56 \mathrm{~mm}$ \\
\hline SNA & $85,09^{\circ}$ \\
\hline SNB & $83,28^{\circ}$ \\
\hline ANB & $1,81^{\circ}$ \\
\hline Angulo interincisivos & $133,51^{\circ}$ \\
\hline .NA & $22,26^{\circ}$ \\
\hline -NA & $4,34 \mathrm{~mm}$ \\
\hline -NB & $4,54 \mathrm{~mm}$ \\
\hline IMPA & $83,37^{\circ}$ \\
\hline NB & $22,42^{\circ}$
\end{tabular}

Foi realizado o índice de Pont-Korklaus ${ }^{x}$ para avaliar a necessidade de expansão ou disjunção da maxila. Foram medidas mesiodistais dos incisivos superiores permanentes que apresentam a soma de $31 \mathrm{~mm}$. Foi medida a largura 4/4 e 6/6 e comparado com valores da tabela utilizando o padrão facial do paciente que é mesofacial (Tabela 1 e 2), pode-se concluir que o paciente necessitava expansão maxilar. O paciente apresentava dentadura permanente completa até segundos molares erupcionados e todos os terceiros molares em formação, maloclusão Classe I de Angle, mordida cruzada unilateral esquerda de primeiro pré-molar a segundo molar, distoversão do dente 12, linguoversão do 22, infravestibuloversão do 13 e 23, mesioversão do 32 e 43 (Figura 2). A largura dos arcos superior e inferior foram medidas com paquímetro digital Digimatic Coliper (Mitutoyo Sul Americana, Kawasaki, Japão nos modelos em gesso.

Foi proposto tratamento com o aparelho quadrihélice para descruzamento da mordida posterior, assim, foram instalados separadores na mesial e distal dos primeiros molares superiores. Após uma semana foi realizada a seleção de bandas para o 16 e 26, brunidura, soldagem de tubos vestibulares e moldagem de transferência. Após sete dias foi instalado o aparelho com seguinte protocolo: isolamento relativo com abridor de boca, rolete de algodão e sugador, profilaxia e condicionamento ácido com Ácido Fosfórico a 37\% (FGM, Joinville - Santa Catarina, Brasil). Em seguida foi aplicada adesivo fotopolimerizável para esmalte e dentina Ambar (FGM, Joinville - Santa Catarina, Brasil), jato de ar por 5 segundos a uma distância de $\pm 10 \mathrm{~cm}$ e fotopolimerização com fotopolimerizador Radii-Cal (SDI, Victoria, Austrália). Em seguida foi aplicada resina fotopolimerizável Ortho Bite (FGM, Joinville - Santa Catarina, Brasil) no interior das bandas e o aparelho foi colocado em posição, realizado a polimerização.

Tabela 2: Índice de Pont-Korklaus

\begin{tabular}{l|l|l|l}
\hline & Paciente & Padrão facial média & Expansão \\
\hline Distância de 14 e 24 & $32 \mathrm{~mm}$ & $36,7 \mathrm{~mm}$ & $-4,7 \mathrm{~mm}$ \\
\hline Distância de 36 e 46 & $45 \mathrm{~mm}$ & $47,7 \mathrm{~mm}$ & $-2,7 \mathrm{~mm}$ \\
\hline
\end{tabular}



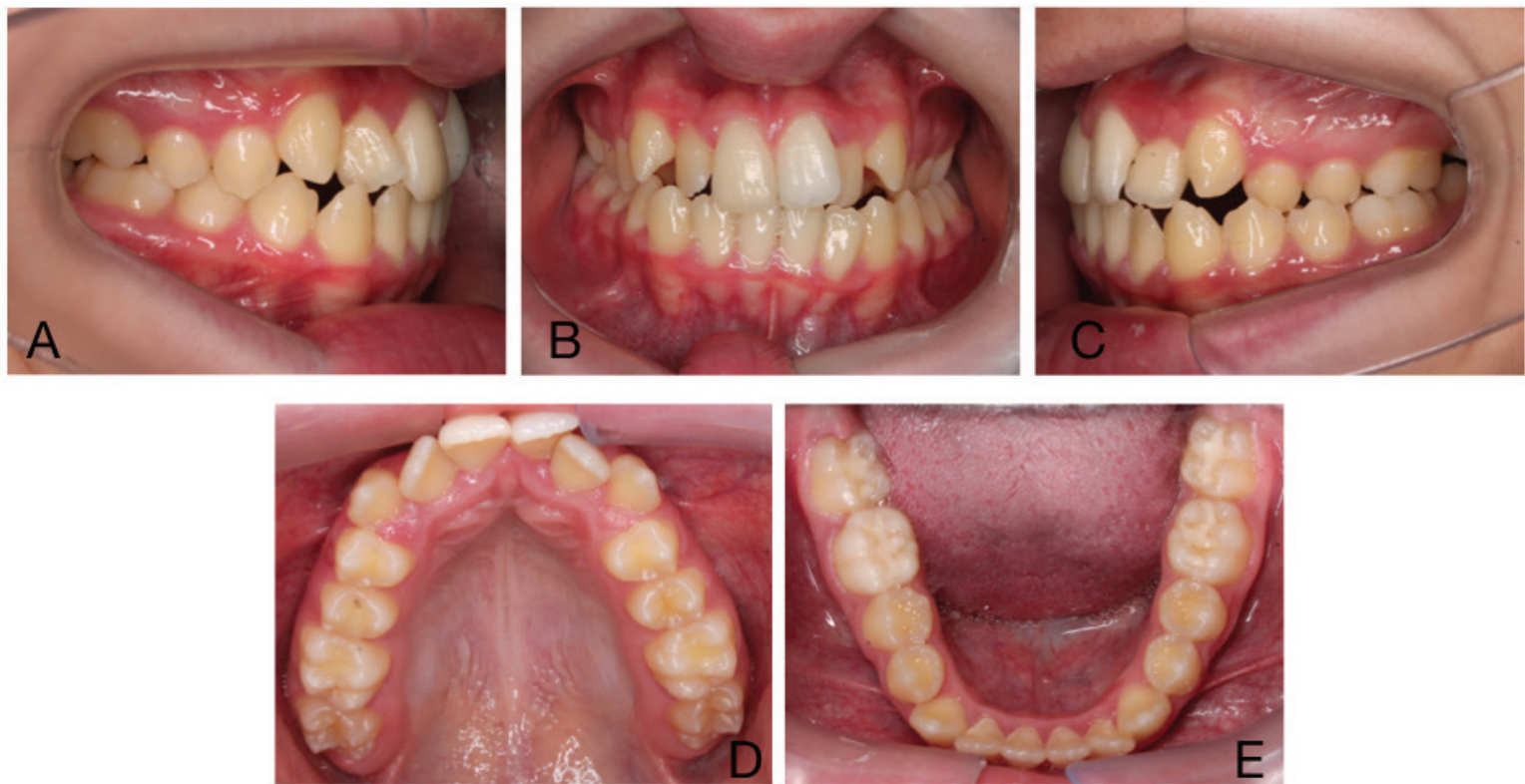

Figura 2: Fotos intrabucais pré-tratamento (A-E).

O aparelho manteve-se passivo após sua cimentação por 70 dias devido a evasão do paciente por motivos particulares. Quando retornou à consulta foi realizada ativação com alicate tridente (Quinelato, Rio Claro - São Paulo, Brasil) no braço esquerdo. Novas ativações foram realizadas após 21 dias. Passados 42 dias a ativação foi realizada no braço em região do 24 e 25 (esses dentes permaneciam cruzados e os demais já haviam sido descruzados com sobrecorreção). Após 28 dias foi feita uma nova ativação do braço em região do 24 e 25 (Figura 3).
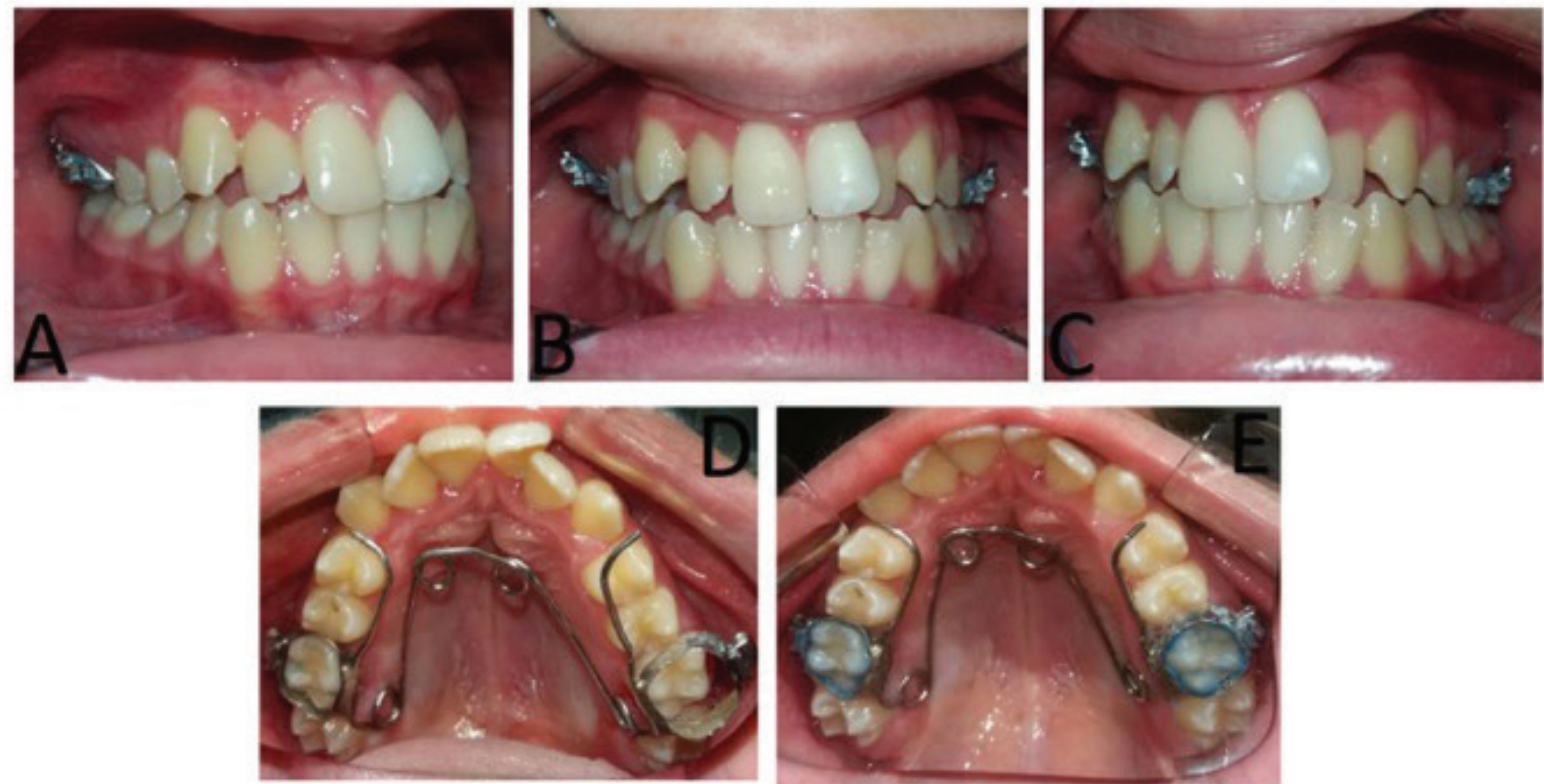

Figura 3: Fotos após 5 meses da instalação (A-E). Conferência da quantidade de ativação do braço em região de 24 e 25 previamente à cimentação (D). Aparelho cimentado (E). 
Depois de 28 dias observou-se o descruzamento total de primeiro pré-molar a segundo molar, e manteve-se o aparelho desativado como forma de contenção. Por conta da evasão do paciente foi feita uma avaliação após 56 dias, deixando o aparelho desativado como contenção (Figura 4). O paciente foi encaminhado para instalação de aparelho ortodôntico fixo.
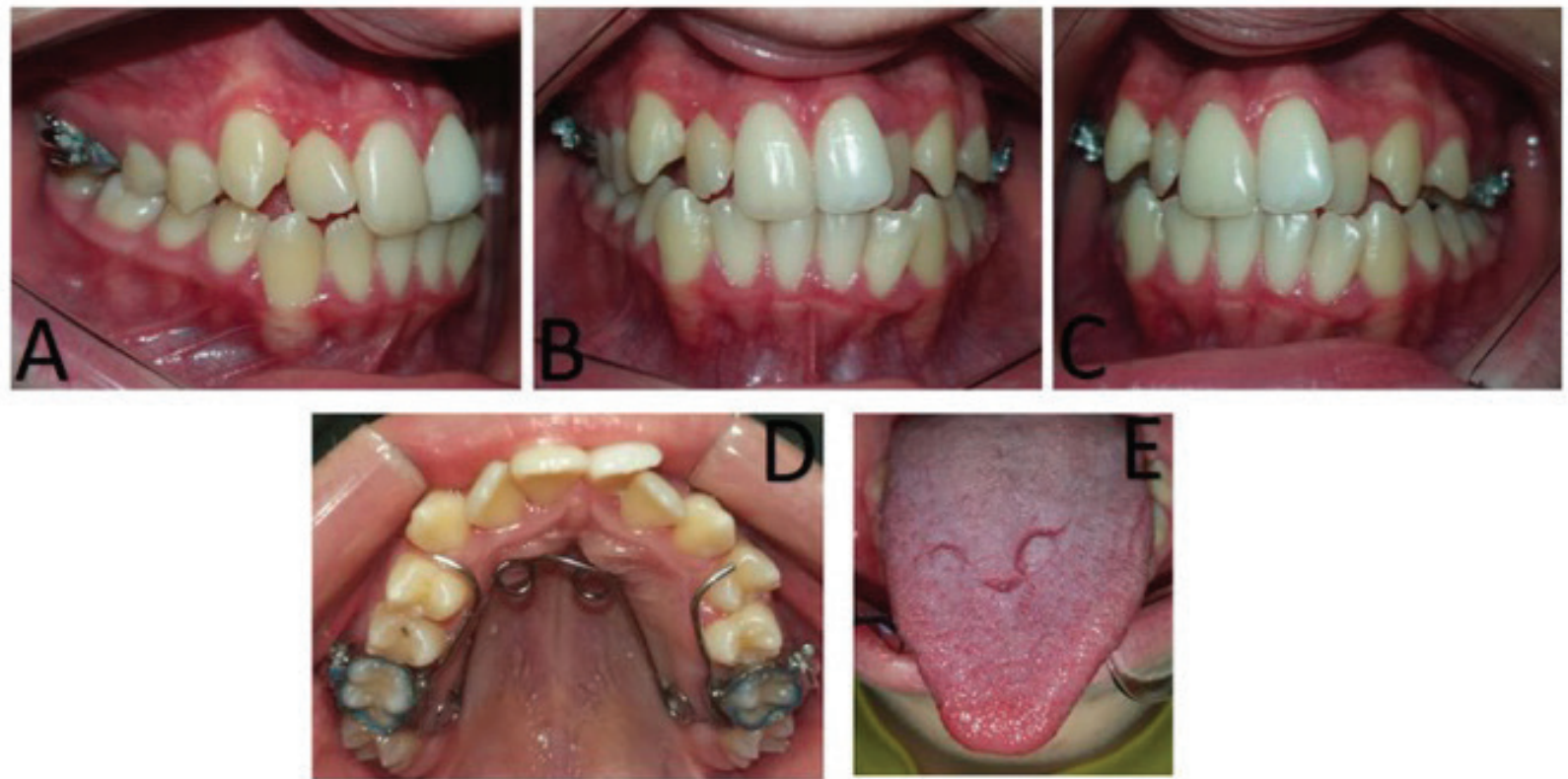

Figura 4: Fotografias após 8 meses da instalação (A-E). Descruzamento total (A-D). Manutenção do aparelho desativado como contenção (D). Língua com marca da hélice do aparelho (E).

Ao término do tratamento foi possível observar alterações na dimensão transversal dos arcos e em outros parâmetros (Tabela 3).

Tabela 3: informações antes da instalação do aparelho quadrihélice e após o descruzamento dos dentes de primeiro pré-molar a segundo molar.

\begin{tabular}{l|l|l}
\hline & T0 $(\mathrm{mm})$ & T1 $(\mathrm{mm})$ \\
\hline Discrepância de modelos superiores & -8 & -7 \\
\hline Discrepância de modelos inferiores & -3 & -1 \\
\hline Sobremordida & 2,46 & 3,33 \\
\hline Sobressaliência & 2,08 & 1,58 \\
\hline Distância de 13 e 23 & 33,76 & 33,76 \\
\hline Distância de 14 e 24 & 37,02 & 45,12 \\
\hline Distância de 15 e 25 & 43,94 & 47,96 \\
\hline Distância de 16 e 26 & 49,73 & 53,79 \\
\hline Distância de 33 e 43 & 26,79 & 25,91 \\
\hline Distância de 34 e 44 & 34,88 & 33,98 \\
\hline Distância de 35 e 45 & 40,45 & 39,86 \\
\hline Distância de 36 e 46 & 43,62 & 44,38 \\
\hline
\end{tabular}




\section{DISCUSS ÃO}

Considera-se que o objetivo do tratamento do paciente G.F.W.L.S. foi alcançado com sucesso: relação tranversal adequada entre maxila e mandibula e correção da mordida cruzada unilateral esquerda após a utilização do aparelho quadrihélice.

O quadrihélice convencional consiste em um par de hélices anteriores, unidos por um fio que é a ponte anterior. O fio entre as hélices anterior e posterior denomina-se ponte palatina. As extremidades de fios livres adjacentes às hélices posteriores são os braços exteriores. Podem ser incorporadas modificações ao aparelho como barra transpalatina ou dobrada para frente, ${ }^{13}$ grade palatina ${ }^{13,14}$, acrílico unilateral ou mola digital, entre outros ${ }^{14}$.

Esse aparelho também pode ser utilizado para expansão da sutura palatina ${ }^{13,15}$, a desvantagem é que seu atrito com a mucosa pode desencadear granulomas piogênicos ${ }^{16}$. No caso do paciente G.F.W.L.S observou-se a marca do aparelho na região dorsal da língua, pois o aparelho ocupa um espaço que anteriormente era apenas dela.

A literatura relata casos de mordida cruzada posteiror dentoalveolar finalizado com sucesso utilizando o aparelho quadrihélice $e^{2-4,6,8,12,13,15,17,18}$, corroborando com o que foi observado nesse estudo. Também há resultados satisfatórios da correção da mordida cruzada posterior dentoalveolar com outros aparelhos, inclusive removivel ${ }^{3,6,17}$. O quadrihélice pode ser indicado tanto para crianças como para adultos ${ }^{17}$ sendo que a média de idade é de 8 a 18 anos $^{2-4,6,8,9,13,18}$. Se a alteração transversal for acompanhada por uma vertical ou maloclusão anterior-posterior, a alteração transversal deve ser tratada primeiro ${ }^{5,9}$; assim primeiro foi introduzido o aparelho quadrihélice para posteriormente utilizar o aparelho fixo no paciente.

Quando a alteração transversal for esquelética o tratamento deve ser feito assim que os primeiros molares permanentes superiores erupcionarem. Sabe-se que o tratamento precoce é essencial para um resultado estável sendo indicada a disjunção maxilar ${ }^{5,9}$, remoção de interferências oclusais e eliminação de hábitos parafuncionais ${ }^{9}$. Este tratamento visa proporcionar uma melhor relação dente/base óssea, assim melhorando a função mastigatória e estabelecendo uma relação simétrica dos côndilos e fossa mandibular ${ }^{1}$.

Quando comparados os efeitos do tratamento com quadrihélice e aparelho removível a um grupo controle sem mordida cruzada, observou-se que houve um aumento transversal após o uso dos aparelhos, porém o tempo de tratamento com quadrihélice foi de 7,7 meses e com removivel 12,5 meses. Observou-se que na mandíbula as distâncias intercaninos e intermolares permaneceram quase inalteradas indicando que utilizando o aparelho superior para expansão quase não há alteração transversal no arco inferior, indicando um aparelho removível inferior se necessário ${ }^{6}$. No caso relatado, em que o paciente tem a dentição permanete completa, houve diminução de largura na região de caninos e pré-molares e aumento de $0,76 \mathrm{~mm}$ na região intermolares do arco inferior, talvez pela retroposição de língua devido ao espaço ocupado no palato. A literatura relata como vantagem do aparelho quadrihélice a correção espontânea da largura mandibular concomitante à expansão do arco maxilar na dentição mista ${ }^{18}$. Estudo que avaliou a expansão lenta com disjuntores haas e hyrax, aparelhos removíves e quadrihélice observaram que não houve diferença estatiscamente significante na expansão dentoalveolar independente do tipo de aparelho utilizado ${ }^{20}$. 
Observa-se com o uso do quadrihélice efeitos de inclinação dentária uma vez que a correção é dentoalveolar. A largura intercaninos e intermolares é geralmente aumentada devido a inclinação das coroas dentarias para vestibular ${ }^{5}$, observa-se um aumento médio entre as distâncias intercaninos de 0,95, de intermolares de 0,96 a 0,993,15. No caso G.F.W.L.S. observou-se um aumento entre as distancias intercaninos, interpremolares e intermolares.

A média do tempo de tratamento para correção da mordida cruzada posterior com o quadrihélice é de 6 semanas a 1 ano $^{2-4,6,8,9,18}$, do paciente G.F.W.L.S. foi um periodo de 8 meses, dentro da faixa de tempo preconizada na literatura. A ativação do aparelho pode ser feito de forma intraoral e extra-oral ${ }^{14,18}$ uma vez por mês ${ }^{3,18}$. Optou-se pela ativação extra-oral no caso relatado a cada mês devido a facilidade de manuseo do aparelho sem deformação dos braços e pela facilidade de inserção e apoio nos molares, verificando precisamente a quantidade de ativação, além disso quando feito de forma extra-oral esse tipo de ativação causa um decréscimo da força original.

A expansão será considerada suficiente quando houver uma sobrecorreção de 2 a $3 \mathrm{~mm}$ ou quando a superfície oclusal da cúspide lingual superior do molar entrar em contato com a vertente oclusal da cúspide vestibular do molar inferior ${ }^{14}$. Depois de corrigir a maloclusão, deve-se permanecer com contenção $\mathrm{o}^{3,5,9,14}$ até os dentes permanentes substituírem os dentes decíduos ${ }^{5}$. O período de contenção varia de 2 a 4 meses e é feito com o próprio aparelho desativado ${ }^{3,14}$ Em conclusão, o aparelho quadrihélice foi eficaz na correção da mordida cruzada dentoalveolar unilateral.

\section{CONCLUSÃO}

O aparelho quadrihélice foi eficaz na correção da mordida cruzada dento alveolar unilateral.

\section{REFERÊNCIAS}

1. Almeida RRd, Almeida MRd, Oltramari-Navarro PVP, Conti ACdCF, Navarro RdL, Marques HVA. Posterior crossbite-treatment and stability. J Appl Oral Sci. 2010: 20(2): 286 - 294.

2. Sahad MdG, Grieco FAD, Cartaxo ZBP, Pinto EG, Prokopowitsch I, Araki ÂT. Tratamento da má oclusão de Classe II, subdivisão direita, segundo a terapia bioprogressiva. Rev Clín Ortod Dental Press. 2012; 11(1): 92-7.

3. Godoy F, Godoy-Bezerra J, Rosenblatt A. Treatment od posterior crossbite comparing 2 appliances: A community-based trial. Am J Orthod Dentofacial Orthop. 2011; 139(1).

4. Huynh T, Kennedy DB, Joondeph DR, Bollen AM. Treatment response and stability old slow maxillary expansion using Haas, hyrax, and quad-helix appliances: A retrospective study. Am J Orthod Dentofacial Orthop. 2009; 136(3): 331-9.

5. Peiro AC. Interceptive orthodontics: The need for early diagnosis and treatment od posterior crossbites. Med Oral Patol Oral Cir Bucal. 2006; 11(2): 210-4.

6. Bjerklin K. Follow-Up Control of Patients with Unilateral Posterior Cross-Bite Treated with Expansion Plates or the Quad-Helix Appliance. J Orofac Orthop. 2000; 61(2): 112-24.

7. Almeida MRd, Pereira ALP, Almeida RRd, Almeira-Pedrin RRd, Silva Filho OGd. Prevalence of malocclusion in children aged 7 to 12 years. Dental Press J Orthod. 2011; 16(4): 226-31.

8. Vinay C, Sandeep V, Hanumanth Rao CH, Uloopi KS, Silva Kumar A. Modified quad helix appliaance for thumb sucking and cross bite correction. Contemp Clin Dent. 2013; 4(4): 523-6. 
9. Yaseen SM, Acharya R. Hexa Helix: Modified Quad Helix Appliance to Correct Anterior and Posterior Crossbites in Mixed Dentition. Case Reports in Dentistry - Hindawi Publishing Corporation. 2012.

10. Duarte MS. O aparelho quadrihélice (Quad-helix) e suas variações. R Dental Press Ortodon Ortop Facial. 2006; 11(2): 128-156.

11. Mucedero M, Franchi L, Giuntini V, Vangelisti A, McNamara Jr JA, Cozza P. Stability of quad-helix/crib therapy in dentoskeletal open bite: A long-term controlled study. Am J Orthod Dentofacial Orthop. 2013; 5: 695-703.

12. Santos R, Pithon M. Avaliação mecânica do aparelho quadri-hélice baixo teor de níquel. Dental Press J Orthod. 2013; 18(3): 35-8.

13. Prakash A, Tandur AP, Rai S. Slow Expansion In Cleft Patient With Quad-Helix. Indian J Dent Adv. 2012; 4(1): 772-5.

14. Raveli DB, Aguiar JVM, Sampaio LP, Soares PRC. Quadr-hélice: partes constituintes, forma de construção e ativação. Revista da Associação Paulista de Especialistas em Ortodontia - Ortopedia Facial. 2004; 2(2): 93-103. 15. Aizenbud D, Ciceu C, Rachmiel A, Hazan-Molina H. Reverse Quad Helix Appliance: Differential Anterior Maxillary Expansion od the Cleft Area Before Bone Grafting. J Craniofac Surg. 2012; 23(5): 440-3.

16. Acharya PN, Gill D, Lloyd T. Pyogenic granuloma: a rare side complication from an orthodontic appliance. J Orthod. 2011; 38:(4) 290-3.

17. O'Neill J. Quad-helix appliances may be more successful than removable expansion plates at correcting posterior crossbites. Evid Based Dent. 2015; 16(1): 25-6.

18. Shundo I, Kobayashi Y, Endo T. Short-term treatment effects of quad-helix on maxillomandibular expansion in patients with maxillary incisor crowding. Odontology. 2012; 100(1): 76-86.

19. Almeida MAdO, Quintão CCA, Brunharo IHVP, Koo D, Coutinho BR. A correção da mordid cruzada posterior unilateral com desvio funcional melhora a assimetria facial? R Dental Press Ortodon Ortop Facial. 2009; 14(2): 89-94.

20. Sandikçioglu M, Hazar S. Skeletal and dental changes after maxillary expansion in the mixed dentition. Am J Orthod Dentofacial Orthop. 1997; 111(3): 321-7. 